\title{
Interatomic-distance dependence of resonant energy-transfer phenomena
}

\author{
F. Grüll, A. B. Voitkiv, and C. Müller \\ Institut für Theoretische Physik I, Heinrich Heine Universität Düsseldorf, Universitätsstrasse 1, 40225 Düsseldorf, Germany
}

(Received 7 April 2020; accepted 6 August 2020; published 25 August 2020)

\begin{abstract}
It is well known that interatomic or intermolecular interactions driven by two-center electronic dipole-dipole correlations fall off rapidly with the intersite distance. We show, however, that the effective strength of interatomic reaction channels, which are triggered by a resonant field, can exhibit a nonmonotonous distance dependence, being strongly reduced when the atoms come closer. This surprising result is demonstrated by considering resonant two-center photoionization as an example. Our findings are supported by available experimental data.
\end{abstract}

DOI: 10.1103/PhysRevResearch.2.033303

\section{INTRODUCTION}

Interatomic and intermolecular processes have been under very active scrutiny in recent years. The research area has been strongly triggered by the prediction of interatomic Coulombic decay (ICD), where electronic excitation energy of an atom is transferred radiationlessly to a neighbor atom, resulting in its ionization [1-3]. ICD is of particular importance when a single-center Auger decay is energetically forbidden, and can proceed much faster than radiative decay. It was first observed in noble-gas dimers and clusters [4,5]. Corresponding measurements rely on advanced experimental techniques, such as third-generation synchrotron sources and few-body coincidence spectrometers [6].

Similar interatomic energy-transfer processes are known in various areas of physics, comprising exciton dynamics in solids [7], quantum optical ensembles, and cold Rydberg gases [8]. They also play an important role in chemistry and biology, as exemplified by ICD in water [9] and hydrated biomolecules [10], lattice dynamics in polymers [11], and Förster resonances in chromophores [12]. Slow electrons set free via ICD cascades are of great relevance for applied radiation biology [13]. It has thus been concluded that interatomic energy-transfer reactions are ubiquitous in nature.

In most of the cases, the interatomic coupling arises from the long-range interaction between two dipoles. It is of the form (in atomic units)

$$
\hat{V}_{e e}=\frac{\mathbf{r} \cdot \boldsymbol{\xi}}{R^{3}}-\frac{3(\mathbf{r} \cdot \mathbf{R})(\boldsymbol{\xi} \cdot \mathbf{R})}{R^{5}},
$$

with the internuclear separation $\mathbf{R}$ and the coordinates of the active electrons $\mathbf{r}$ and $\mathbf{r}^{\prime}=\mathbf{R}+\boldsymbol{\xi}$. The generic $R^{-3}$ scaling is modified when retardation effects or nondipole transitions are

Published by the American Physical Society under the terms of the Creative Commons Attribution 4.0 International license. Further distribution of this work must maintain attribution to the author(s) and the published article's title, journal citation, and DOI. considered. Yet, always, the interaction quickly falls off when the interatomic distance grows. Accordingly, intersite energy transfers are expected to be the more efficient, the closer the atoms lie together.

An interatomic process involving ICD is two-center resonant photoionization (2CPI) in a heteroatomic system of two atoms, say $A$ and $B$ [14-18]. Here, a neighboring atom $B$ is first resonantly excited by photoabsorption, thereby creating an autoionizing state of the two-center system, which afterwards stabilizes via ICD [2]. The original theory [14,15] assumed, for simplicity, two spatially well-separated atoms with fixed distance vector $\mathbf{R}$. Since the photoabsorption step is included in the treatment, a comparison with the direct photoionization of atom $A$ is feasible. Taking application to $\mathrm{Li}$ as atom $A$ and $\mathrm{He}$ as atom $B$ at an interatomic distance of $10 \AA$ as an example, a relative enhancement of 2 CPI over the direct photoionization of Li by a factor $\simeq 10^{6}$ is predicted.

2CPI was experimentally observed in $\mathrm{NeHe}$ dimers [19-21]. Up to $\mathrm{a} \simeq 60$-fold relative enhancement was found, which is very substantial, though much smaller than the enormous amplification predicted for the LiHe model system. This discrepancy appears astonishing in light of the fact that the internuclear separation in the $\mathrm{NeHe}$ ground state is substantially less than in LiHe lying in the range $\approx 2.5-6 \AA$ [22]. Accordingly, ICD proceeds much faster in $\mathrm{NeHe}$ than in LiHe, occurring on a timescale of hundreds of femtoseconds. A considerable enhancement of photoionization due to 2CPI has very recently also been seen in ArNe clusters [23], but again at a much lower scale than in $\mathrm{LiHe}$.

The reasons for so vastly different (and counterintuitive) levels of enhancement have not been clarified yet. It is suggestive to assume that the differences between the measurements and the original theory result from the molecular structure of the target systems which was not taken into account there. In fact, ICD in NeHe and other noble-gas dimers is known to be very sensitive to the vibrational nuclear motion [22,24].

However, as we show in this paper, the difference between the relative enhancement predicted in LiHe versus the one observed in NeHe is not caused by the molecular structure of a dimer in the first place. The reduction in 2CPI efficiency 
rather occurs because the $\mathrm{Ne}$ and $\mathrm{He}$ atoms are so close to each other. As will be seen below, this is not a particular feature specific to the $\mathrm{NeHe}$ system, but reflects a general behavior of the enhancement with respect to the intersite separation. Standing in sharp contrast to the intuitive expectation that close distances should generally facilitate interatomic energy-transfer processes [see Eq. (1)], this result offers an unexpected perspective on two- (and multi)center phenomena.

\section{THEORETICAL DESCRIPTION}

We consider a system of two atoms, $A$ and $B$, which are initially in their ground states. They are separated by a sufficiently large distance $R$ (covering at least several $\AA$ ), such that their individuality is basically preserved, and exposed to a resonant electromagnetic field of the form

$$
\mathbf{F}(t)=F_{0} \cos (\omega t) \mathbf{e}_{z},
$$

which is taken in the dipole approximation. Here, $\omega$ is the angular frequency and $F_{0}$ is the field amplitude.

To start with, we assume the atoms to be at rest and take the position of the nucleus of atom $A$ as the origin and denote the coordinates of the nucleus of atom $B$, the (active) electron of atom $A$ and that of atom $B$, by $\mathbf{R}, \mathbf{r}$, and $\mathbf{r}^{\prime}=\mathbf{R}+\boldsymbol{\xi}$, respectively, where $\boldsymbol{\xi}$ is the position of the electron of atom $B$ with respect to its nucleus. Let atom $B$ have an excited state $\chi_{e}$ reachable from the ground state $\chi_{g}$ by a dipole-allowed transition.

The total Hamiltonian describing the two atoms in the external electromagnetic field reads

$$
\hat{H}=\hat{H}_{0}+\hat{V}_{e e}+\hat{W},
$$

where $\hat{H}_{0}$ is the sum of the Hamiltonians for the noninteracting atoms $A$ and $B$, and $\hat{V}_{e e}$ is the interaction between the atoms. $\hat{W}=\hat{W}_{A}+\hat{W}_{B}=\mathbf{F}(t) \cdot\left(\mathbf{r}+\mathbf{r}^{\prime}\right)$ denotes the interaction of the atoms with the electromagnetic field in the length gauge. It is assumed that $\omega_{g e} R / c \ll 1$, where $\omega_{g e}$ is the atomic transition frequency and $c$ is the speed of light, such that retardation effects can be neglected.

In the 2CPI process, one has essentially three different basic two-electron configurations, which are schematically illustrated in Fig. 1: (I) $\Phi_{i}=\varphi_{g}(\mathbf{r}) \chi_{g}(\boldsymbol{\xi})$, with total energy $E_{i}=$ $\varepsilon_{g}+\epsilon_{g}$, where both atoms are in the corresponding ground states $\varphi_{g}$ and $\chi_{g}$; (II) $\Phi_{a}=\varphi_{g}(\mathbf{r}) \chi_{e}(\xi)$, with total energy $E_{a}=$ $\varepsilon_{g}+\epsilon_{e}$, in which atom $A$ is in the ground state, while atom $B$ is in the excited state $\chi_{e}$; (III) $\Phi_{f}=\varphi_{\mathbf{p}}(\mathbf{r}) \chi_{g}(\boldsymbol{\xi})$, with total energy $E_{f}=\varepsilon_{p}+\epsilon_{g}$, where the electron of atom $A$ has been emitted into the continuum with asymptotic momentum $\mathbf{p}$, while the electron of atom $B$ has returned to the ground state.

Within the second order of time-dependent perturbation theory, the probability amplitude for 2CPI can be written as

$$
\begin{aligned}
S_{\mathbf{p}}^{(2)}= & -\int_{-\infty}^{\infty} d t\left\langle\Phi_{f}\left|\hat{V}_{e e}\right| \Phi_{a}\right\rangle e^{-i\left(E_{a}-E_{f}\right) t} \\
& \times \int_{-\infty}^{t} d t^{\prime}\left\langle\Phi_{a}\left|\hat{W}_{B}\right| \Phi_{i}\right\rangle e^{-i\left(E_{i}-E_{a}\right) t^{\prime}} .
\end{aligned}
$$

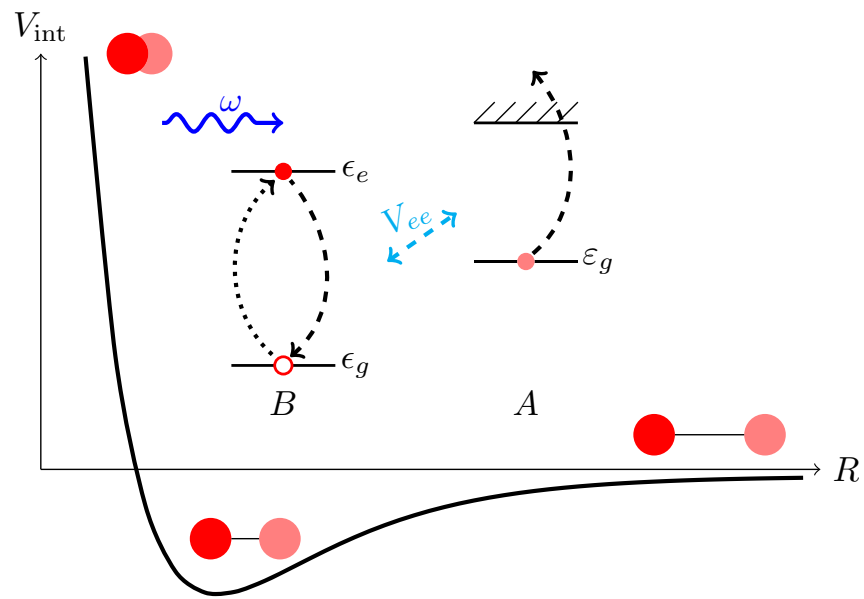

FIG. 1. Scheme of two-center resonant photoionization (2CPI), embedded in a generic potential curve $V_{\text {int }}(R)$ of a van der Waals dimer $A-B$. First, atom $B$ is resonantly photoexcited; its subsequent decay by ICD leads to the ionization of atom $A$ via an interatomic dipole-dipole interaction $V_{e e}$. The shape of the potential curve determines at which internuclear distance $R$ the process mostly occurs.

Performing the time integrations, we obtain

$$
\begin{aligned}
S_{\mathbf{p}}^{(2)}= & -i \pi\left\langle\varphi_{\mathbf{p}}|\mathbf{r}| \varphi_{g}\right\rangle \cdot\left(\mathbf{e}_{z}-\frac{3 R_{z}}{R^{2}} \mathbf{R}\right) \\
& \times \frac{F_{0}}{R^{3}} \frac{\left|\left\langle\chi_{e}\left|\xi_{z}\right| \chi_{g}\right\rangle\right|^{2}}{\Delta+\frac{i}{2} \Gamma} \delta\left(\varepsilon_{p}-\varepsilon_{g}-\omega\right),
\end{aligned}
$$

where the detuning from the resonance, $\Delta=\epsilon_{g}+\omega-\epsilon_{e}$, has been introduced and the total width $\Gamma=\Gamma_{r}+\Gamma_{a}$ of the excited state $\chi_{e}$ in atom $B$ inserted. It accounts for the finite lifetime of this state and consists of the radiative width $\Gamma_{r}$ and the two-center Auger (ICD) width $\Gamma_{a}$ [25]. The $\delta$ function in Eq. (5) displays the law of energy conservation in the process.

From the transition amplitude, we can obtain the total ionization cross section in the usual way by taking the absolute square, integrating it over the photoelectron momentum, and dividing it by the interaction time $\tau$ and the incident flux $j=\frac{c F_{0}^{2}}{8 \pi \omega}$, that is,

$$
\begin{aligned}
\sigma_{\mathrm{at}}^{(2)}(R)= & \frac{1}{j \tau} \int \frac{d^{3} p}{(2 \pi)^{3}}\left|S_{\mathbf{p}}^{(2)}\right|^{2} \\
= & \int d \Omega_{p}\left|\left\langle\varphi_{\mathbf{p}}|\mathbf{r}| \varphi_{g}\right\rangle \cdot\left(\mathbf{e}_{z}-3 \cos \theta_{R} \mathbf{e}_{R}\right)\right|^{2} \\
& \times \frac{\omega p}{2 \pi c R^{6}} \frac{\left|\left\langle\chi_{e}\left|\xi_{z}\right| \chi_{g}\right\rangle\right|^{4}}{\Delta^{2}+\frac{1}{4} \Gamma^{2}} .
\end{aligned}
$$

Here, the value of $p$ is fixed by the energy conservation and we have introduced the unit vector $\mathbf{e}_{R}=\mathbf{R} / R$ along the internuclear separation and the angle $\theta_{R}$ between $\mathbf{R}$ and the field direction [26]. Equation (6) can be rewritten using the cross section $\sigma_{A}^{(1)}=\int d \Omega_{p}\left|\left\langle\varphi_{\mathbf{p}}|z| \varphi_{g}\right\rangle\right|^{2} \frac{\omega p}{2 \pi c}$ for the direct photoionization of atom $A$ by the electromagnetic field. For the special cases, when the separation vector $\mathbf{R}$ between the atoms $A$ and $B$ is oriented either along the field direction 
or perpendicular to it, we obtain

$$
\begin{aligned}
\sigma_{\mathrm{at}}^{(2)}(R) & =\frac{\alpha^{2}}{R^{6}} \frac{\left|\left\langle\chi_{e}\left|\xi_{z}\right| \chi_{g}\right\rangle\right|^{4}}{\Delta^{2}+\frac{1}{4} \Gamma^{2}} \sigma_{A}^{(1)} \\
& =\left(\frac{3 \alpha c^{3}}{4 \omega_{g e}^{3} R^{3}}\right)^{2} \frac{\Gamma_{r, g e}^{2}}{\Delta^{2}+\frac{1}{4} \Gamma^{2}} \sigma_{A}^{(1)},
\end{aligned}
$$

where $\alpha=2$ for $\mathbf{R} \| \mathbf{F}_{0}$ and $\alpha=1$ for $\mathbf{R} \perp \mathbf{F}_{0}$. In the second step, the dipole matrix element has been expressed by the corresponding radiative width, $\Gamma_{r, g e}=\frac{4 \omega_{g e}^{3}}{3 c^{3}}\left|\left\langle\chi_{e}\left|\xi_{z}\right| \chi_{g}\right\rangle\right|^{2}$, with $\omega_{g e}=\epsilon_{e}-\epsilon_{g}$. The compact formula (7) applies to two individual atoms at a distance $R$, carrying a single active electron each.

Below we will consider diatomic systems containing helium as atom $B$. In this case, the two equivalent electrons must be described by appropriately symmetrized wave functions and their interaction with the field by a two-particle extension of the operator $\hat{W}_{B}$. Assuming that the field is exactly resonant $(\Delta=0)$, the ratio of the 2CPI and direct photoionization cross sections becomes

$$
\frac{\sigma_{\mathrm{at}}^{(2)}(R)}{\sigma_{A}^{(1)}}=\left(\frac{3 \alpha c^{3}}{2 \omega^{3} R^{3}}\right)^{2} \frac{\Gamma_{r, g e}^{2}}{\left(\Gamma_{r}+\Gamma_{a}\right)^{2}} .
$$

By expressing the Auger width $\Gamma_{a}$ according to

$$
\Gamma_{a}(R)=\frac{3 \alpha^{2}}{8 \pi} \frac{c^{4}}{\omega^{4} R^{6}} \Gamma_{r, g e} \sigma_{A}^{(1)}
$$

(see, e.g., [27]), Eq. (7) can be put in a form which enables one to have a better understanding of the interatomic distance dependence of the $2 \mathrm{CPI}$,

$$
\sigma_{\text {at }}^{(2)}(R)=2 \sigma_{B}^{(\mathrm{exc})} \frac{\Gamma_{a} \Gamma_{r, g e}}{\left(\Gamma_{r}+\Gamma_{a}\right)^{2}},
$$

where $\sigma_{B}^{(\mathrm{exc})}=3 \pi c^{2} / \omega^{2}[28]$ is the cross section for resonant photoexcitation of atom $B$.

Apart from a numerical prefactor of the order of unity, Eq. (10) can be represented as a product of two terms, $\sigma_{B}^{(\mathrm{exc})} \Gamma_{r, g e} /\left(\Gamma_{r}+\Gamma_{a}\right)$ and $\Gamma_{a} /\left(\Gamma_{r}+\Gamma_{a}\right)$.

The first of them describes the photoexcitation step of 2CPI resulting in the creation of the intermediate state. Since $\Gamma_{r, g e} /\left(\Gamma_{r}+\Gamma_{a}\right)<\Gamma_{r, g e} / \Gamma_{r}$, it is seen that compared to an isolated atom $B\left(\Gamma_{a}=0\right)$, the probability for the resonant excitation in the $A-B$ system is reduced due to a broadening of the resonance caused by the presence of the additional deexcitation pathway via ICD.

The second term, which is simply a branching ratio, determines the probability that afterwards the intermediate state decays via ICD. Unlike the first one, it increases with $\Gamma_{a}$ approaching 1 at $\Gamma_{a} \gg \Gamma_{r}$.

At $\Gamma_{r, g e} \simeq \Gamma_{r}$ (which, in particular, holds for the systems studied below), the optimal enhancement of 2CPI over direct photoionization is reached for $\Gamma_{a} \approx \Gamma_{r}$, strongly decreasing both at $\Gamma_{a} \ll \Gamma_{r}$ and $\Gamma_{a} \gg \Gamma_{r}$. Since $\Gamma_{a}$ falls with $R$, this suggests a nonmonotonous behavior of 2CPI on the dimer size: there is an "optimal" value where the efficiency of 2CPI is maximal and from where it decreases not only towards larger, but also towards smaller sizes.
We now include the effects of the nuclear motion in a weakly bound molecule. The Coulomb, exchange, and van der Waals interactions between the atoms $A$ and $B$ create a static potential $V_{\text {int }}(R)$, whose form depends on the electron configuration and in which the atomic nuclei occupy discrete vibrational levels. The wave functions of the system are accordingly amended, $\Psi_{i, a, f}=\Phi_{i, a, f}(\mathbf{r}, \boldsymbol{\xi}) \psi_{i, a, f}^{(v)}(R)$, to include the internuclear coordinate. When the derivation given above is repeated with these molecular states, the ratio of cross sections adopts the modified form [18]

$$
\frac{\sigma_{\mathrm{mol}}^{(2)}}{\sigma_{\mathrm{mol}}^{(1)}}=\frac{\sigma_{\mathrm{at}}^{(2)}\left(R_{\mathrm{eq}}\right)}{\sigma_{A}^{(1)}}\left(\frac{R_{\mathrm{eq}}^{3} \mathrm{FC}_{i, a}\left\langle\psi_{f}^{\left(v_{f}\right)}\left|R^{-3}\right| \psi_{a}^{\left(v_{a}\right)}\right\rangle}{\mathrm{FC}_{i, f}}\right)^{2},
$$

for a given set of vibrational quantum numbers $v_{i}, v_{a}$, and $v_{f}$. Here, the Franck-Condon factors,

$$
\mathrm{FC}_{i, a}=\left\langle\psi_{a}^{\left(v_{a}\right)} \mid \psi_{i}^{\left(v_{i}\right)}\right\rangle=\int d R\left[\psi_{a}^{\left(v_{a}\right)}(R)\right]^{*} \psi_{i}^{\left(v_{i}\right)}(R),
$$

and similarly for $\mathrm{FC}_{i, f}$ have been introduced. The quadratic factor in Eq. (11), which accounts for the nuclear motion, will be referred to as $F_{\text {nuc }}$ below. Note that it was made dimensionless by inserting the equilibrium distance $R_{\text {eq }}$ between the nuclei into the formula.

The quantum number $v_{a}$ in Eq. (11) determines the vibrational nuclear wave function of the two-center autoionizing state and fixes the precise value of the associated resonant transition energy. The full expression for $\sigma_{\text {mol }}^{(2)}$ contains a coherent sum over the intermediate state and an incoherent sum over the final-state quantum numbers, including $v_{f}[18]$.

Before proceeding further, we note that the processes of 2CPI and direct photoionization are generally subject to quantum interference because they lead to the same final state (atom $B$ merely serves as a catalyzer). However, for parameters where 2CPI strongly dominates, the interference is of minor importance and may be neglected.

\section{DISCUSSION}

Based on Eqs. (8), (10), and (11), we can now compare the relative enhancement of $2 \mathrm{CPI}$ over direct photoionization in $\mathrm{LiHe}$ and $\mathrm{NeHe}$ dimers.

For a system of $\mathrm{Li}$ and $\mathrm{He}$ at $R=10 \AA$, considered as individual atoms, the ratio $\sigma_{\text {at }}^{(2)} / \sigma_{A}^{(1)}$ in Eq. (8) attains the value $\approx 10^{6}$, assuming that the incident photon energy $\omega \approx 21.2 \mathrm{eV}$ is resonant to the $1 s \rightarrow 2 p$ transition in helium and taking $\alpha=1$ for definiteness. In this scenario, the radiative width $\Gamma_{r}^{(2 p)}=\Gamma_{r, g e}^{(2 p)} \approx 1.18 \times 10^{-6} \mathrm{eV}$ [29] is much larger than the Auger width $\Gamma_{a}$. When a LiHe dimer [30] is considered instead, the single resonance splits into a multiplet of resonances, in accordance with the various vibrational transitions [18]. On each of these resonances, the nuclear motion tends to reduce the enhancement, but not dramatically $\left(F_{\text {nuc }} \sim 0.1\right.$ for favored transitions $v_{i} \rightarrow v_{a} \rightarrow v_{f}$ ). Averaging over the molecular orientations with respect to the field, when the dimers are randomly distributed, leads to a further reduction of the cross section by a geometrical factor of the order of unity [31]. We point out that the resulting enhancement $\sim 10^{5}$ refers to the top of the $2 \mathrm{CPI}$ resonance peak. 
In the experiment [19], $\mathrm{Ne}$ is ionized by synchrotron photons of energy $\omega \approx 23.1 \mathrm{eV}$, which corresponds to the $1 s \rightarrow 3 p$ transition in He (note that the $1 s \rightarrow 2 p$ transition energy lies below the ionization potential of $\mathrm{Ne}$ ). The largest enhancement (by a factor of $\simeq 60$; see [19]) was observed when the intermediate state $1 s 3 p \pi$ with $v_{a}=2$ was populated. The Auger width $\Gamma_{a}$ of this state can be estimated from the "local" width (9), which applies to a fixed value of $R$, by taking an average over the probability density $\left|\psi_{a}^{\left(v_{a}\right)}(R)\right|^{2}$ of the vibrational state and, accordingly, amounts to $\Gamma_{a} \sim 1 \mathrm{meV}$ [32]. This estimated value of $\Gamma_{a}$ is in reasonable agreement with the results of advanced quantum chemical calculations [20,21]. $\Gamma_{a}$ turns out to be orders of magnitude larger than the radiative width $\Gamma_{r}^{(3 p)} \approx 3.7 \times 10^{-7} \mathrm{eV}[29,33]$.

With these numbers, we obtain $\bar{\sigma}_{\text {mol }}^{(2)} / \sigma_{\text {mol }}^{(1)} \approx 80$, which is 3-4 orders of magnitude smaller than in LiHe. As before, we have set $\alpha=1$ since the field component perpendicular to the molecular axis is responsible for $\pi$-state excitation. $\bar{\sigma}_{\text {mol }}^{(2)}$ involves an average over the molecular orientations in the gas target and, accordingly, amounts to $2 / 3$ of the cross section at $\theta_{R}=\frac{\pi}{2}$. Besides, the nuclear-motion factor was estimated as $F_{\text {nuc }} \approx 1$ because the transitions mainly occur in a small range of internuclear distances around the equilibrium value.

At this point, we already see that the enhancement is much smaller than in LiHe: in a NeHe dimer, $\Gamma_{a}$ is huge compared to $\Gamma_{r}$, and the increase of the branching ratio to essentially 1 cannot compensate for the very strong decrease in the excitation probability caused by a very large broadening of the resonance due to the ICD channel. Therefore, it is this interplay between the photoexcitation and decay steps of 2CPI [see Eq. (10)] which is the key in explaining the counterintuitive result that the relative enhancement of photoionization due to 2CPI can be much weaker in a relatively small $\mathrm{NeHe}$ system compared to a large LiHe dimer.

We further note that the applied synchrotron beam in the experiment on $\mathrm{NeHe}$ [19] was not monochromatic, but consisted of an incoherent superposition of frequency components with a spectral width of $\Delta \omega=1.7 \mathrm{meV}$, which effectively broadens the resonance. At $\min \left\{\Gamma_{a}, \Delta \omega\right\} \gg \Gamma_{r}$, this effect can be approximately taken into account by the replacement $\Gamma^{2} \rightarrow \Gamma_{a}\left(\Gamma_{a}+\Delta \omega\right)$ in the denominator of Eq. (10). Accordingly, it leads to a damping of 2CPI in NeHe by roughly a factor of $\Gamma_{a} /\left(\Gamma_{a}+\Delta \omega\right) \approx 0.37$. This reduces the calculated ratio to $\bar{\sigma}_{\text {mol }}^{(2)} / \sigma_{\text {mol }}^{(1)} \simeq 30$, which is to be compared with an approximately 60-fold enhancement observed in the experiment [19].

We point out that from the measured data, a larger ICD width of $\Gamma_{a} \approx 2-2.5 \mathrm{meV}$ was deduced in [19]. It leads to a somewhat smaller ratio of $\bar{\sigma}_{\mathrm{mol}}^{(2)} / \sigma_{\mathrm{mol}}^{(1)} \approx 20$.

2CPI in NeHe dimers has recently been studied with a focus on the angular distribution of emitted electrons [21]. To this end, $a b$ initio electronic structure and nuclear dynamics calculations were performed, taking the interference with the direct photoionization channel into account. In particular, apart from angular emission spectra, a total cross section of
2CPI with excitation of the $1 s 3 p \pi\left(v_{a}=2\right)$ state of approximately $270 \mathrm{Mb}$ was reported, when a photon beam width of about $1 \mathrm{meV}$ was accounted for. Our present estimates for this system complement the numerical calculations of [21] by providing additional physical insights, which help one to better understand the origin of the observed level of enhancement in $\mathrm{NeHe}$.

The above discussion indicates that the spectral width might have a large detrimental impact on the enhancement effect. However, unlike the ICD width $\Gamma_{a}$, this factor can be avoided by using coherent light sources with a very high degree of monochromaticity. Indeed, the feasibility of atomic spectroscopy at $\omega \lesssim 20 \mathrm{eV}$ and megahertz bandwidths $\left(\Delta \omega \lesssim 10^{-7} \mathrm{eV}\right)$ has been demonstrated by extending the frequency-comb technique into the extreme ultraviolet domain [34]. Such sources can be employed for an experimental observation of the predicted huge enhancement of photoionization in LiHe. If a larger bandwidth of $\Delta \omega \approx 10^{-5} \mathrm{eV}$ $\left(\Delta \omega \approx 10^{-3} \mathrm{eV}\right.$ ) was applied instead, the level of enhancement would be reduced by roughly a factor of $\Gamma_{r} /\left(\Gamma_{r}+\Delta \omega\right)$ and amount to $\bar{\sigma}_{\text {mol }}^{(2)} / \sigma_{\text {mol }}^{(1)} \sim 10^{4}\left(\sim 10^{2}\right)$, accordingly.

\section{CONCLUSION}

We have shown that the resonant enhancement of photoionization due to two-center dipole-dipole correlations can be very strongly reduced when the intersite distance decreases, even though the strength of the correlations, per se, greatly increases. This counterintuitive result also applies to other resonant two-center phenomena, such as, for instance, interatomic photo double ionization [35] and two-center dielectronic recombination $[15,36]$, which represents the inverse of 2CPI. All this shows that in order to "extract" the most efficiency from the resonant two-center coupling, the latter must not be too strong, i.e., the interacting centers should not be located too close to each other.

While our study has focused on small diatomic systems, the conclusions are expected to be of general relevance for resonant intersite energy-transfer processes. Their efficiency is optimized at intermediate distances where the decay properties of the single catalyzing center and the whole two(or multi)center system are balanced. This main result has potentially far-reaching consequences, given the omnipresence of interatomic and intermolecular processes in nature. In particular, it can help to predict and classify in which physical, chemical and biological systems these energy-transfer phenomena are most efficient.

\section{ACKNOWLEDGMENTS}

This work has been funded by the Deutsche Forschungsgemeinschaft (DFG, German Research Foundation) under Grant No. 349581371 (MU 3149/4-1 and VO 1278/4-1). Useful conversations with R. Dörner on the experimental conditions of Ref. [19] are gratefully acknowledged.
[1] L. S. Cederbaum, J. Zobeley, and F. Tarantelli, Phys. Rev. Lett. 79, 4778 (1997).
[2] While the initial state for ICD is usually prepared by photoionization of an inner-valence electron, so-called resonant ICD 
occurs after photoexcitation. See, e.g., K. Gokhberg, A. B. Trofimov, T. Sommerfeld, and L. S. Cederbaum, Europhys. Lett. 72, 228 (2005); K. Gokhberg, V. Averbukh, and L. S. Cederbaum, J. Chem. Phys. 124, 144315 (2006).

[3] For reviews on ICD, see U. Hergenhahn, J. Electron Spectrosc. Relat. Phenom. 184, 78 (2011); V. Averbukh et al., ibid. 183, 36 (2011); T. Jahnke, J. Phys. B 48, 082001 (2015).

[4] S. Marburger, O. Kugeler, U. Hergenhahn, and T. Möller, Phys. Rev. Lett. 90, 203401 (2003).

[5] T. Jahnke, L. Foucar, J. Titze, R. Wallauer, T. Osipov, E. P. Benis, A. Alnaser, O. Jagutzki, W. Arnold, S. K. Semenov et al., Phys. Rev. Lett. 93, 083002 (2004); Y. Morishita et al., ibid. 96, 243402 (2006).

[6] J. Ullrich, R. Moshammer, A. Dorn, R. Dörner, L. P. H. Schmidt, and H. Schmidt-Böcking, Rep. Prog. Phys. 66, 1463 (2003).

[7] J. Frenkel, Phys. Rev. 37, 17 (1931); G. D. Scholes and G. Rumbles, Nat. Mater. 5, 683 (2006).

[8] T. Amthor, M. Reetz-Lamour, S. Westermann, J. Denskat, and M. Weidemüller, Phys. Rev. Lett. 98, 023004 (2007); C. S. E. van Ditzhuijzen, A. F. Koenderink, J. V. Hernández, F. Robicheaux, L. D. Noordam, and H. B. van Linden van den Heuvell, ibid. 100, 243201 (2008).

[9] T. Jahnke et al., Nat. Phys. 6, 139 (2010); M. Mucke et al., ibid. 6, 143 (2010); C. Richter et al., Nat. Commun. 9, 4988 (2018).

[10] X. G. Ren, E. L. Wang, A. D. Skitnevskaya, A. B. Trofimov, K. Gokhberg, and A. Dorn, Nat. Phys. 14, 1062 (2018).

[11] S. Suhai, Phys. Rev. B 51, 16553 (1995).

[12] T. Förster, Ann. Phys. (Leipzig) 437, 55 (1948); T. Renger, V. May, and O. Kühn, Phys. Rep. 343, 137 (2001); E. A. JaresErijman and T. M. Jovin, Nat. Biotechnol. 21, 1387 (2003).

[13] K. Gokhberg, P. Kolorenč, A. I. Kuleff, and L. S. Cederbaum, Nature (London) 505, 661 (2014).

[14] B. Najjari, A. B. Voitkiv, and C. Müller, Phys. Rev. Lett. 105, 153002 (2010).

[15] A. B. Voitkiv and B. Najjari, Phys. Rev. A 82, 052708 (2010).

[16] J. Peřina, Jr., A. Lukš, W. Leoński, and V. Peřinova, Phys. Rev. A 83, 053416 (2011); V. Peřinová, A. Lukš, J. Křepelka, and J. Peřina, Jr., ibid. 90, 033428 (2014).

[17] A. B. Voitkiv, C. Müller, S. F. Zhang, and X. Ma, New J. Phys. 21, 103010 (2019).

[18] F. Grüll, A. B. Voitkiv, and C. Müller, Phys. Rev. A 102, 012818 (2020).

[19] F. Trinter, J. B. Williams, M. Weller, M. Waitz, M. Pitzer, J. Voigtsberger, C. Schober, G. Kastirke, C. Müller, C. Goihl et al., Phys. Rev. Lett. 111, 233004 (2013).

[20] G. Jabbari, S. Klaiman, Y.-C. Chiang, F. Trinter, T. Jahnke, and K. Gokhberg, J. Chem. Phys. 140, 224305 (2014).

[21] A. Mhamdi, F. Trinter, C. Rauch, M. Weller, J. Rist, M. Waitz, J. Siebert, D. Metz, C. Janke, G. Kastirke et al., Phys. Rev. A 97, 053407 (2018).

[22] N. Sisourat, H. Sann, N. V. Kryzhevoi, P. Kolorenč, T. Havermeier, F. Sturm, T. Jahnke, H.-K. Kim, R. Dörner, and L. S. Cederbaum, Phys. Rev. Lett. 105, 173401 (2010).

[23] A. Hans, P. Schmidt, C. Ozga, C. Richter, H. Otto, X. Holzapfel, G. Hartmann, A. Ehresmann, U. Hergenhahn, and A. Knie, J. Phys. Chem. Lett. 10, 1078 (2019).

[24] Signatures of the nuclear dynamics have also been identified in ICD in He dimers. See T. Havermeier, T. Jahnke, K. Kreidi, R. Wallauer, S. Voss, M. Schöffler, S. Schössler, L. Foucar, N. Neumann, J. Titze et al., Phys. Rev. Lett. 104, 133401 (2010); N. Sisourat, N. V. Kryzhevoi, P. Kolorenč, S. Scheit, and L. S. Cederbaum, Phys. Rev. A 82, 053401 (2010); A. Mhamdi, J. Rist, T. Havermeier, R. Dörner, T. Jahnke, and P. V. Demekhin, ibid. 101, 023404 (2020).

[25] Since we treat 2CPI in the second order of perturbation theory, the width $\Gamma$ of the excited state in atom $B$ must be added by "hand." Both the radiative and two-center widths of the excited state appear automatically if, for instance, a nonrelativistic (noncovariant) QED approach is employed to treat the process [15].

[26] Note that Eq. (6) contains an effective polarization vector $\mathbf{n}_{\mathrm{eff}}=$ $\mathbf{e}_{z}-3 \cos \theta_{R} \mathbf{e}_{R}$, which depends on the field polarization and the relative interatomic orientation. It may prove useful for the interpretation of angular emission patterns from 2CPI [21].

[27] F. Grüll, A. B. Voitkiv, and C. Müller, Phys. Rev. A 100, 032702 (2019).

[28] B. H. Bransden and C. J. Joachain, Physics of Atoms and Molecules (Longman Group, Harlow, 1990).

[29] Atomic spectra database of the National Institute of Standards and Technology (NIST), available at https://www.nist.gov/pml/ atomic-spectra-database (unpublished).

[30] B. Friedrich, Physics 6, 42 (2013).

[31] ICD in helium droplets doped with lithium and rubidium atoms has recently been observed in L. Ben Ltaief et al., J. Phys. Chem. Lett. 10, 6904 (2019). In this system, ICD was found to occur predominantly from the $1 s 2 s^{1} S$ state in helium and to compete with a charge-exchange decay channel similar to Penning ionization.

[32] Since the typical interatomic distance amounts to 5-12 Bohr radii, the dipole interaction (1) is well suited to describe ICD in $\mathrm{NeHe}$, following $1 s \rightarrow 3 p$ excitation in helium (see, also, [20]). The next order of the multipole expansion would be suppressed, accordingly, by about one order of magnitude.

[33] The $1 s 3 p$ state in He decays radiatively to the $1 s^{2}\left(\Gamma_{r, g e}^{(3 p)}\right)$ and $1 s 2 s$ states. However, the contribution to $\Gamma_{r}^{(3 p)}$ from the latter is smaller than $\Gamma_{r, g e}^{(3 p)}$ by a factor of about 50 .

[34] A. Cingöz, D. C. Yost, T. K. Allison, A. Ruehl, M. E. Fermann, I. Hartl, and J. Ye, Nature (London) 482, 68 (2012).

[35] A. C. LaForge, M. Shcherbinin, F. Stienkemeier, R. Richter, R. Moshammer, T. Pfeifer, and M. Mudrich, Nat. Phys. 15, 247 (2019); A. Eckey, A. B. Voitkiv, and C. Müller, J. Phys. B 53, 055001 (2020).

[36] C. Müller, A. B. Voitkiv, J. R. Crespo López-Urrutia, and Z. Harman, Phys. Rev. Lett. 104, 233202 (2010). 